\title{
Short Communication: Potential tests of plant growth bacteria for the control of Peronosclerospora philipinensis in corn
}

\author{
NURASIAH DJAENUDDIN, SYAFRUDDIN, BAHARUDDIN PATANDJENGI ${ }^{\boldsymbol{}}$, TUTIK KUSWINANTI \\ Department of Plant Pests and Diseases, Faculty of Agriculture, Universitas Hasanuddin. Jl. Perintis Kemerdekaan Km. 10, Tamalanrea, Makassar \\ 90245, South Sulawesi, Indonesia. Tel./fax.: +62-411-586014, `email: nurasiahdjaenuddin@ pasca.unhas.ac.id, v baharunhas@yahoo.com
}

Manuscript received: 16 May 2020. Revision accepted: 31 July 2020

\begin{abstract}
Djaenuddin N, Syafruddin, Patandjengi B, Kuswinanti T. 2020. Short Communication: Potential tests of plant growth bacteria for the control of Peronosclerospora philipinensis in corn. Biodiversitas 21: 3886-3892. The study was conducted at the Laboratory and Screen House of the Indonesian Cereals Research Institute (ICERI). The stages of the study were (i) potential test of bacterial isolates that have the ability to control downy mildew disease in vivo in corn and (ii) molecular identification of the selected bacterial isolates. Experiments were arranged in a Completely Randomized Design (CRD) with treatment of 24 bacteria which suspected to be growth-promoting bacteria. The parameters observed were disease intensity, percent disease suppression, plant height, chlorophyll content, and crop wet weight. The result showed that only five bacterial isolates namely, Bacillus albus strain MCCC 1A02146, Bacillus cereus strain IAM 12605, Bacillus paramycoides strain MCCC 1A04098, Pseudomonas stutzeri strain CCUG 11256, Serratia marcescens subsp. sakuensis strain KRED, have the ability to induce resistance to downy mildew disease caused by Peronosclerospora philipinensis.
\end{abstract}

Keywords: Corn, disease incidence, Downey mildew, induce resistance, Peronosclerospora philipinensis

\section{INTRODUCTION}

Downy mildew (DM) is one of the most harmful diseases of corn caused by Peronosclerospora philipinensis (Lantican et al. 2013). Of the 12 species of DM in corn, only three have been recorded as the most destructive species, namely $P$. sorghi, $P$. phillipinensis, and $P$. maydis. More research so far has focused on $P$. sorghi and $P$. philipinensis (Lukman et al. 2016). DM can cause crop damage up to $100 \%$, especially on susceptible varieties, and markedly reduce the corn productivity in Indonesia (Pudjiwati et al. 2013). Synthetic pesticides are mostly used to control the disease. In addition to rising economic costs, improper control by synthetic pesticides also negatively impacts environmental sustainability (Meena et al. 2015).

Synthetic pesticides are able to suppress plant diseases, but damage beneficial microorganisms that are found in the soil and also pollute the soil environment (Tariq et al. 2017). To overcome the negative effects of the use of synthetic pesticides, it is necessary to have eco-friendly controls that produce healthy and high-quality plants. A possible alternative to control DM is eco-friendly plant growth-promoting rhizobacteria or PGPR.

In some studies, PGPR such as Bacillus cereus, $B$. amyloliquefaciens, B. substilis, B. pasteurii, B. pumilus, $B$. mycoides, and $B$. sphaericus have been used to control disease incidence and severity from various diseases in various plants (Resti et al. 2013). The use of Bacillus sp. can suppress DM on grapes caused by Plasmopora viticola (Zhang et al. 2017). However, detailed information about the ability of bacteria in controlling DM in corn is still limited. According to Mota et al. (2017), it is very important to develop a fast and efficient method for selecting bio-control microorganisms, especially when evaluating a large number of bacterial isolates. The discovery of bacterial isolates suitable for the treatment of corn seeds in controlling $P$. philipinensis is very significant in the field of biological control and development of biopesticide production in Indonesia. The aim of this study was to obtain bacterial isolates capable of controlling DM disease, stimulate the corn growth and molecular identification of these isolates.

\section{MATERIALS AND METHODS}

\section{Study area}

The research was carried out in the laboratory and screen house of ICERI at Maros from September to December 2019. A total of 24 bacterial isolates were isolated from various agricultural and plantations habitats of the South Sulawesi (Table 1).

\section{Bacterial potential test in controlling downy mildew in vivo}

\section{Seed treatment}

Maize seeds were soaked in selected bacterial suspensions) with a concentration of $10^{9} \mathrm{cfu}^{-1}$ for two hours and then dried. For comparison, K1 with one type of synthetic pesticides (ai; metallaxyl) and $\mathrm{K} 2$ ie seeds soaked with sterile distilled water. 
Table 1. Diversity of bacterial isolates collected from various agricultural and plantation habitats

\begin{tabular}{lc}
\hline Name of isolates & Name of collection sites \\
\hline Ms-3 & Maros \\
Ms-4 & Maros \\
Ms- 8 & Maros \\
Be-2.1 & Bone \\
Be-3 & Bone \\
Be-4 & Bone \\
Wo-2.1 & Wajo \\
Wo-2.2 & Wajo \\
Wo-3.1 & Wajo \\
Wo-3.2 & Wajo \\
Ga-1.2 & Gowa \\
Ga-1.3 & Gowa \\
Ga-2 & Gowa \\
Ga-2.1 & Gowa \\
Ga-2.2 & Gowa \\
Ga-3 & Gowa \\
Ga-3.1 & Gowa \\
Si-1 & Sinjai \\
Si-3 & Sinjai \\
Si-4 & Sinjai \\
Bg-1 & Bantaeng \\
Bg-1.2 & Bantaeng \\
Tp-2 & Tanjung Pinang \\
Tp-3.1 & Tanjung Pinang \\
\hline
\end{tabular}

\section{Planting}

Seeds were planted in micro plots (with an area of $5 \mathrm{~m}^{2}$ ) in the screen house with a spacing of $75 \mathrm{~cm} \times 25 \mathrm{~cm}$ with one seed per hole, so that there are 20 plants per row in one replication. The seeds used were Anoman variety. After being planted, at the age of 7-10 days after planting (DAP), spore inoculation was carried out by spraying a conidia suspension of $P$. philippinensis $\left(10^{6}\right.$ spores $\left./ \mathrm{ml}\right)$ on the leaves of corn plants at around 03.00-04.00 am. Inoculation was repeated three days later to get optimal symptoms. Urea fertilizer was applied as much as $150 \mathrm{~kg} / \mathrm{ha}$ at 10 days after planting (DAP).

\section{Molecular identification of bacterial isolates}

Molecular identification was performed on selected bacterial isolates which have a potential to control DM disease in corn. Bacterial DNA was isolated and purified using the Quick-DNA тм Fungal/Bacterial Miniprep Kit (D6005). The isolated DNA was then amplified using a pair of primers 27F (5'AGAGTTTGATCCTGGCTCAG 3') and primers $1492 \mathrm{R}$ (5' TACGGYTACCTTGTTACGA CTT 3 '). The conditions of PCR were as follows: set at a temperature of initial denaturation at $95^{\circ} \mathrm{C}$ for $1 \mathrm{~min}$, followed by 35 cycles of denaturation at $95^{\circ} \mathrm{C}$ for 15 seconds, annealing at $55^{\circ} \mathrm{C}$ for 15 seconds and finally extension at $72^{\circ} \mathrm{C}$ for 42 seconds. The PCR result was then electrophoresed using MyTaq HS Red Mix (Bioline) and visualized under UV Transilluminator. Amplification of DNA from selected isolates produced a band that was \pm $1400 \mathrm{bp}$. The sequencing of DNA was performed at Genetics Science Inc. Co. in Jakarta, Indonesia. Data sequencing results were matched with the Gene Bank NCBI using the BLAST on http://www.ncbi.nlm.nih.org.

\section{Observation and data collection}

Observations were done every day for three weeks to determine the incubation period of downy mildew. Other variables observed were $(\%)$ disease incidence at $21,28,35$ DAP, percentage of disease suppression, and plant height (21 and 35 DAP). For chlorophyll, content leaves were sampled at the base, middle, and tip portions on 35 DAP, and the wet weight of corn plants was recorded at 49 DAP. Disease incidence was calculated according to the formula (Sekarsari et al. 2013):

$$
\mathrm{DI}(\%)=\frac{\mathrm{A}}{\mathrm{B}} \times 100
$$

Where;

DI : DM incidence (\%)

A : Number of DM-infected plants

B : Number of plants observed

Disease suppression percentage in disease incidence (DI) was determined according to the following equation:

Disease suppression $\%=\{(\mathrm{DI}$ in control \%-DI in treatment \%)/DI in control \} x 100

\section{Research design and data analysis}

The experiments were arranged in a completely randomized design with the treatment of 24 bacterial isolates and 2 controls. The experiment was carried out in two replicates. The data were analyzed using one-way analysis of variance followed by LSD test at 5\% significance level.

\section{RESULTS AND DISCUSSION}

\section{Bacterial potential test in controlling downy mildew in vitro}

The result showed that application of bacteria on seeds has a positive effect on the rate of germination. In some bacterial treatments namely Ga-1, Ga-2.2, Ga-3, Ms-8, Wo2.1, Wo-3.1, and Wo-3.2 germination reaches up to $100 \%$, whereas in the controls (K1 and K2) germination was $<100 \%$. Symptoms of downy mildew began to appear at 17 days after planting in the controls and bacterial treatments (Table 2).

Result of in vivo test of 24 bacterial isolates can be seen in Table 3. The incidence of DM in aqua control (K2) at 28 and 35 DAP was $52.8 \%$ and $89.7 \%$ respectively, while it was $44.1 \%$ and $59.1 \%$ in synthetic control (K1). The observation of the percentage of downy mildew infection starting 21 and 28 DAP has not been significant between bacterial treatments and control. However, at 35 DAP, twelve bacterial isolates had significantly lower disease intensity $<7 \%$ with infection conditions in control of $89 \%$.

All isolates were able to suppress downy mildew infection, indicating the average effectiveness of disease suppression as compared to control. The effectiveness of disease suppression ranges from $2-100 \%$. The treatment of six bacterial isolates i.e. $\mathrm{Bg}-1, \mathrm{Ga}-2, \mathrm{Ga}-3, \mathrm{Si}-4, \mathrm{Tp}-2$, and 
Tp-3.1 was found to be effective in controlling downy mildew disease and even symptoms did not appear at 35 DAP, which means that these isolates have the ability to suppress downy mildew disease up to $100 \%$. Bg-1.2, Ga2.1, Ga-2.2, Ga-3.1, and $\mathrm{Si}-3$ were the other bacterial isolates, with infection rate ranging from 2.9 to $6.3 \%$ and suppression rate between 88 and 97\% (Figure 1).

The effect of bacterial isolates on the growth of corn plants can be seen in Table 4. Results from 24 bacterial isolates showed that they did not have a negative effect on plant growth and average plant height was not significantly different from controls. While in many isolates, chlorophyll content was significantly different from the controls. This was related to the rate of downy mildew infection in each treatment. Among the 24 bacterial isolates, the highest chlorophyll content was recorded in Si-4 (43), followed by Ga-3.1 (41.9), Si-3 (41.2), Ga-2.1 and Ga-2 each (41.0), $\mathrm{Tp}-2$ and $\mathrm{Bg}-1$ each (40.9) and $\mathrm{Si}-1$ (40.8), whereas in aqua control and synthetic control (K1and K2) it was 24.7 and 29 respectively. The highest $5.48 \mathrm{~kg}$ wet weight was recorded in $\mathrm{Si}-1$, followed by $5.37 \mathrm{~kg}$ in $\mathrm{Ga}-3$ and $5.15 \mathrm{~kg}$ in $\mathrm{Ga}-2.1$ bacterial isolate. While wet weight was $3.20 \mathrm{~kg}$ and $3.27 \mathrm{~kg}$ in aqua control (K2) and synthetic control (K1) respectively. The six isolates that were able to suppress the infection significantly had higher wet weight than controls. It was also recorded that not all the bacterial isolates were capable to trigger plant growth, as their results were lower than controls.

\section{Identification of bacterial isolates}

$\mathrm{Six}$ isolates namely $\mathrm{Bg}-1, \mathrm{Ga}-2, \mathrm{Ga}-3, \mathrm{Si}-4, \mathrm{Tp}-2$, and Tp-3.1 were selected for molecular identification as they found to be effective in DM suppression. Amplification of DNA from 6 selected isolates produced a band that was \pm $1400 \mathrm{bp}$. After the sequencing of nucleic acid and BLAST analysis, the isolated identities were obtained as in Table 5. Phylogenetic analysis and the alignment of $16 \mathrm{~s}$ rDNA gene sequences based on the 16s rDNA gene library was shown in Figure 2.

Results of BLAST analysis of Bg-1 isolate had $99.7 \%$ similarity with Bacillus albus strain MCCC 1A02146, Ga-2 and $\mathrm{Si}-4$ isolates had $99 \%$ similarity with Bacillus cereus strain IAM 12605, Ga-3 isolates had $99.8 \%$ similarity with Bacillus paramycoides strain MCCC 1A04098, Tp-2 isolates had $99.8 \%$ similarity with Pseudomonas stutzeri strain CCUG 11256 and Tp-3.1 isolate had 99.6\% similarity with Serratia marcescens subsp. sakuensis strain KRED (Table 5).

Seed treatment is thought to systemically protect plants from pathogenic infections so as to reduce the incidence of disease at the beginning of planting. The lowest disease incidence was recorded in $\mathrm{Bg}-1, \mathrm{Ga}-2, \mathrm{Ga}-3, \mathrm{Si}-4, \mathrm{Tp}-2$, and Tp-3.1 isolates which were significantly different from controls. These were the six best isolates to suppress the disease severity of downy mildew on maize, which was significantly different from controls and other treatments.
Table 2. Percentage of seed germination and incubation period of downy mildew in different treatments

\begin{tabular}{lcl}
\hline Treatment & $\begin{array}{l}\text { Germination } \\
(\%)\end{array}$ & $\begin{array}{l}\text { Incubation period } \\
\text { (days) }\end{array}$ \\
\hline Be-2.1 & 94 & $18 \mathrm{abc}$ \\
$\mathrm{Be}-3$ & 93 & $18.5 \mathrm{abc}$ \\
$\mathrm{Be}-4$ & 98 & $18.5 \mathrm{abc}$ \\
$\mathrm{Bg}-1$ & 90 & $0-\mathrm{c}$ \\
$\mathrm{Bg}-1.2$ & 94 & $31.5 \mathrm{a}$ \\
Ga-1.2 & 91 & $18.5 \mathrm{abc}$ \\
$\mathrm{Ga}-1.3$ & 98 & $19.5 \mathrm{abc}$ \\
$\mathrm{Ga}-2$ & 81 & $0-\mathrm{c}$ \\
Ga-2.1 & 100 & $14 \mathrm{abc}$ \\
Ga-2.2 & 100 & $14 \mathrm{abc}$ \\
Ga-3 & 100 & $0-\mathrm{c}$ \\
Ga-3.1 & 90 & $21 \mathrm{abc}$ \\
Ms-3 & 88 & $17 \mathrm{abc}$ \\
Ms-4 & 92 & $17 \mathrm{abc}$ \\
Ms-8 & 100 & $18 \mathrm{abc}$ \\
Si-1 & 95 & $28 \mathrm{ab}$ \\
Si-3 & 97 & $21 \mathrm{abc}$ \\
Si-4 & 92 & $0-\mathrm{c}$ \\
Tp-2 & 95 & $0-\mathrm{c}$ \\
Tp-3.1 & 94 & $0-\mathrm{c}$ \\
Wo-2.1 & 100 & $17 \mathrm{abc}$ \\
Wo-2.2 & 94 & $19 \mathrm{abc}$ \\
Wo-3.1 & 100 & $18 \mathrm{abc}$ \\
Wo-3.2 & 100 & $20.5 \mathrm{abc}$ \\
K1/synthetic fungicide & 97 & $6 \mathrm{bc}$ \\
K2/aquadest & 95 & $5 \mathrm{bc}$ \\
The numbers in the same column followed by the same letter are \\
not significantly different according to the LSD test at $\alpha=0.05$
\end{tabular}

Table 3. Development of downy mildew disease in various DAP in different treatments

\begin{tabular}{|c|c|c|c|}
\hline \multirow{2}{*}{ Treatments } & \multicolumn{3}{|c|}{ DM incidence (\%) at } \\
\hline & 21 DAP & 28 DAP & 35 DAP \\
\hline Be-2.1 & $2.2^{\mathrm{ab}}$ & $46.7^{\text {bcde }}$ & $69.6^{\text {defg }}$ \\
\hline $\mathrm{Be}-3$ & $14.3^{\mathrm{abc}}$ & $54.8^{\mathrm{bc}}$ & $81.3^{\mathrm{efg}}$ \\
\hline Be-4 & $3.7^{\mathrm{ab}}$ & $28.1^{\text {abcde }}$ & $52.6^{\text {def }}$ \\
\hline Bg-1 & $0.0^{\mathrm{a}}$ & $0.0^{\mathrm{a}}$ & $0.0^{\mathrm{a}}$ \\
\hline Bg-1.2 & $2.9^{\mathrm{ab}}$ & $6.3^{\mathrm{ab}}$ & $6.3^{\mathrm{ab}}$ \\
\hline Ga-1.2 & $1.5^{\mathrm{a}}$ & $26.4^{\text {abcde }}$ & $60.8^{\operatorname{defg}}$ \\
\hline Ga-1.3 & $6.9^{a b c}$ & $13.9^{\text {abcd }}$ & $38.5^{\mathrm{bcd}}$ \\
\hline Ga-2 & $0.0^{\mathrm{a}}$ & $0.0^{\mathrm{a}}$ & $0.0^{\mathrm{a}}$ \\
\hline Ga-2.1 & $2.6^{\mathrm{ab}}$ & $5.3^{\mathrm{ab}}$ & $5.3^{\mathrm{ab}}$ \\
\hline Ga-2.2 & $2.8^{a b}$ & $2.8^{\mathrm{ab}}$ & $5.6^{\mathrm{ab}}$ \\
\hline Ga-3 & $0.0^{\mathrm{a}}$ & $0.0^{\mathrm{a}}$ & $0.0^{\mathrm{a}}$ \\
\hline Ga-3.1 & $0.0^{\mathrm{a}}$ & $0.0^{\mathrm{a}}$ & $2.9^{a b}$ \\
\hline Ms-3 & $20.8^{\mathrm{bc}}$ & $60.7^{\mathrm{e}}$ & $87.5^{\text {fg }}$ \\
\hline Ms-4 & $5.1^{\mathrm{abc}}$ & $30.9^{\text {abcde }}$ & $66.3^{\text {defg }}$ \\
\hline Ms-8 & $1.7^{\mathrm{a}}$ & $51.7^{\text {cde }}$ & $72.4^{\text {defg }}$ \\
\hline $\mathrm{Si}-1$ & $5.6^{\mathrm{abc}}$ & $10.8^{a b c}$ & $10.8^{\mathrm{abc}}$ \\
\hline $\mathrm{Si}-3$ & $0.0^{\mathrm{a}}$ & $2.9^{\mathrm{ab}}$ & $2.9^{\mathrm{ab}}$ \\
\hline $\mathrm{Si}-4$ & $0.0^{\mathrm{a}}$ & $0.0^{\mathrm{a}}$ & $0.0^{\mathrm{a}}$ \\
\hline Tp-2 & $0.0^{\mathrm{a}}$ & $0.0^{\mathrm{a}}$ & $0.0^{\mathrm{a}}$ \\
\hline Tp-3.1 & $0.0^{\mathrm{a}}$ & $0.0^{\mathrm{a}}$ & $0.0^{\mathrm{a}}$ \\
\hline Wo-2.1 & $23.7^{c}$ & $44.6^{\text {bcde }}$ & $50.6^{\mathrm{def}}$ \\
\hline Wo-2.2 & $0.0^{\mathrm{a}}$ & $30.0^{\text {abcde }}$ & $45.9^{\text {cde }}$ \\
\hline Wo-3.1 & $6.3^{a b c}$ & $32.2^{\text {abcde }}$ & $67.8^{\text {defg }}$ \\
\hline Wo-3.2 & $2.0^{\mathrm{a}}$ & $23.9^{\text {abcde }}$ & $47.4^{\text {cde }}$ \\
\hline $\mathrm{K} 1 /$ synthetic & $1.6^{\mathrm{a}}$ & $44.1^{\text {bcde }}$ & $59.1^{\text {defg }}$ \\
\hline K2/aquadest & $17.0^{\mathrm{abc}}$ & $52.8^{\text {cde }}$ & $89.7^{\mathrm{g}}$ \\
\hline
\end{tabular}

The numbers in the same column followed by the same letter are not significantly different according to the LSD test at $\alpha=0.05$ 
Table 4. Effect of 24 bacterial isolates on plant height, chlorophyll content and wet weight of corn plants

\begin{tabular}{|c|c|c|c|c|}
\hline \multirow{2}{*}{ Treatment } & \multicolumn{2}{|c|}{ Plant height $(\mathrm{cm})$} & \multirow{2}{*}{$\begin{array}{l}\text { Chlorophyll } \\
\text { content (units) } \\
\text { at } 35 \text { DAP }\end{array}$} & \multirow{2}{*}{$\begin{array}{l}\text { Wet } \\
\text { weight } \\
\text { (kg) }\end{array}$} \\
\hline & 21 DAP & 35 DAP & & \\
\hline Be-2.1 & $72.0^{\mathrm{a}}$ & $123.4^{\mathrm{a}}$ & $28.3^{\mathrm{bcd}}$ & 3. $51^{\mathrm{ab}}$ \\
\hline $\mathrm{Be}-3$ & $71.9^{a}$ & $114.3^{\mathrm{a}}$ & $24.1^{\mathrm{d}}$ & 2. $86^{\mathrm{ab}}$ \\
\hline Be-4 & $74.9^{\mathrm{a}}$ & $123.1^{\mathrm{a}}$ & $28.7^{\mathrm{bcd}}$ & $3.64^{a b c}$ \\
\hline Bg-1 & $71.8^{a}$ & $124.7^{\mathrm{a}}$ & $40.9^{\mathrm{a}}$ & 4. $18^{a b c}$ \\
\hline $\mathrm{Bg}-1.2$ & $69.1^{\mathrm{a}}$ & $129.3^{\mathrm{a}}$ & $39.5^{\mathrm{a}}$ & 4. $37^{\mathrm{ab}}$ \\
\hline $\mathrm{Ga}-1.2$ & $74.5^{\mathrm{a}}$ & $124.4^{\mathrm{a}}$ & $23.7^{\mathrm{d}}$ & $3.73^{a b c}$ \\
\hline Ga-1.3 & $71.8^{a}$ & $123.9^{\mathrm{a}}$ & $30.7^{\mathrm{b}}$ & $3.04^{\mathrm{ab}}$ \\
\hline Ga-2 & $77.0^{\mathrm{a}}$ & $124.3^{\mathrm{a}}$ & $41.0^{\mathrm{a}}$ & $4,30^{\mathrm{ab}}$ \\
\hline Ga-2.1 & $71.1^{\mathrm{a}}$ & $127.0^{\mathrm{a}}$ & $41.0^{\mathrm{a}}$ & 5. $15^{\mathrm{ab}}$ \\
\hline $\mathrm{Ga}-2.2$ & $71.4^{\mathrm{a}}$ & $129.1^{\mathrm{a}}$ & $38.4^{\mathrm{a}}$ & 4. $37^{\mathrm{ab}}$ \\
\hline $\mathrm{Ga}-3$ & $72.2^{\mathrm{a}}$ & $139.8^{\mathrm{a}}$ & $39.4^{\mathrm{a}}$ & $5.37^{\mathrm{a}}$ \\
\hline Ga-3.1 & $73.8^{\mathrm{a}}$ & $126.2^{\mathrm{a}}$ & $41.9^{\mathrm{a}}$ & 4. $40^{\mathrm{ab}}$ \\
\hline Ms-3 & $71.9^{\mathrm{a}}$ & $119.4^{\mathrm{a}}$ & $24.7^{\mathrm{cd}}$ & 3. $20^{\mathrm{ab}}$ \\
\hline Ms-4 & $76.0^{\mathrm{a}}$ & $134.3^{\mathrm{a}}$ & $28.8^{b c d}$ & $3.99 \mathrm{abc}$ \\
\hline Ms-8 & $73.2^{\mathrm{a}}$ & $123.1^{\mathrm{a}}$ & $26.6^{b c d}$ & $3.37 \mathrm{abc}$ \\
\hline $\mathrm{Si}-1$ & $72.9^{a}$ & $129.1^{\mathrm{a}}$ & $40.8^{\mathrm{a}}$ & $5.48^{\mathrm{a}}$ \\
\hline $\mathrm{Si}-3$ & $72.9^{\mathrm{a}}$ & $116.5^{\mathrm{a}}$ & $41.2^{\mathrm{a}}$ & 2. $87^{\mathrm{ab}}$ \\
\hline $\mathrm{Si}-4$ & $66.6^{\mathrm{a}}$ & $131.2^{\mathrm{a}}$ & $43.2^{\mathrm{a}}$ & 4. $32^{a b c}$ \\
\hline Tp-2 & $73.6^{\mathrm{a}}$ & $122.6^{\mathrm{a}}$ & $40.9^{\mathrm{a}}$ & $3.74^{\mathrm{ab}}$ \\
\hline Tp-3.1 & $74.1^{\mathrm{a}}$ & $136.5^{\mathrm{a}}$ & $38.6^{\mathrm{a}}$ & 4. $87^{\mathrm{ab}}$ \\
\hline Wo-2.1 & $67.8^{a}$ & $112.2^{\mathrm{a}}$ & $27.3^{b c d}$ & $2.09^{\mathrm{c}}$ \\
\hline Wo-2.2 & $75.2^{\mathrm{a}}$ & $128.2^{\mathrm{a}}$ & $27.7^{b c d}$ & 2. $66^{\mathrm{bc}}$ \\
\hline Wo-3.1 & $73.5^{\mathrm{a}}$ & $118.0^{\mathrm{a}}$ & $30.1^{b c}$ & $2.98^{\mathrm{ab}}$ \\
\hline Wo-3.2 & $76.7^{\mathrm{a}}$ & $127.8^{\mathrm{a}}$ & $28.1^{\mathrm{bcd}}$ & 3. $45^{\mathrm{ab}}$ \\
\hline $\mathrm{K} 1 /$ synthetic & $70.9^{a}$ & $131.3^{\mathrm{a}}$ & $29.0^{b c d}$ & 4. $00^{\mathrm{ab}}$ \\
\hline $\mathrm{K} 2 /$ aquadest & $64.0^{\mathrm{a}}$ & $115.5^{\mathrm{a}}$ & $24.7^{\mathrm{cd}}$ & $3.27 \mathrm{abc}$ \\
\hline CV $(\%)$ & 7.6 & 10.1 & 7.1 & 28.6 \\
\hline
\end{tabular}

Note: The numbers in the same column followed by the same letter are not significantly different according to the LSD test at $\alpha=0.05$
Table 5. Results of BLAST analysis of bacterial isolates

\begin{tabular}{llc}
\hline $\begin{array}{c}\text { Isolate } \\
\text { code }\end{array}$ & \multicolumn{1}{c}{ Identity } & $\begin{array}{c}\% \\
\text { simila } \\
\text { rity }\end{array}$ \\
\hline $\mathrm{Bg}-1$ & Bacillus albus strain MCCC 1A02146 & 99.74 \\
$\mathrm{Ga}-2$ & Bacillus cereus strain IAM 12605 & 99.93 \\
$\mathrm{Ga}-3$ & Bacillus paramycoides strain MCCC 1A04098 & 99.81 \\
$\mathrm{Si}-4$ & Bacillus cereus strain IAM 12605 & 99.65 \\
$\mathrm{Tp}-2$ & Pseudomonas stutzeri strain CCUG 11256 & 99.86 \\
$\mathrm{Tp}-3.1$ & Serratia marcescens subsp. sakuensis strain KRED & 99.65 \\
\hline
\end{tabular}

\section{Discussion}

The bacterial application had a slower incubation period than the controls, even some bacterial treatments were not affected by the fungus. DMs colonize freely in plants in the absence of other microorganisms that lead to a short incubation period for disease and appearance of early symptoms (Kaur et al. 2011). Plants that have a longer incubation period potentially involving the speed of the plant in activating its defence system which is influenced by the bacteria applied. Using bacterial strains in plants was very important because these microorganisms were able to establish relationships with plants and stimulate plant growth through many beneficial physiological characteristics (Souza et al. 2015). Treatment of antagonistic bacteria can provide a defense system (bioprotectant), as these bacteria secrete antibiotic compounds that were able to signal affected plants. Rhizosphere bacteria act as biological agents through the production of antibiotics, lytic enzymes, hydrogen cyanide, siderophore, or competition for nutrition and space (Raj et al. 2012).

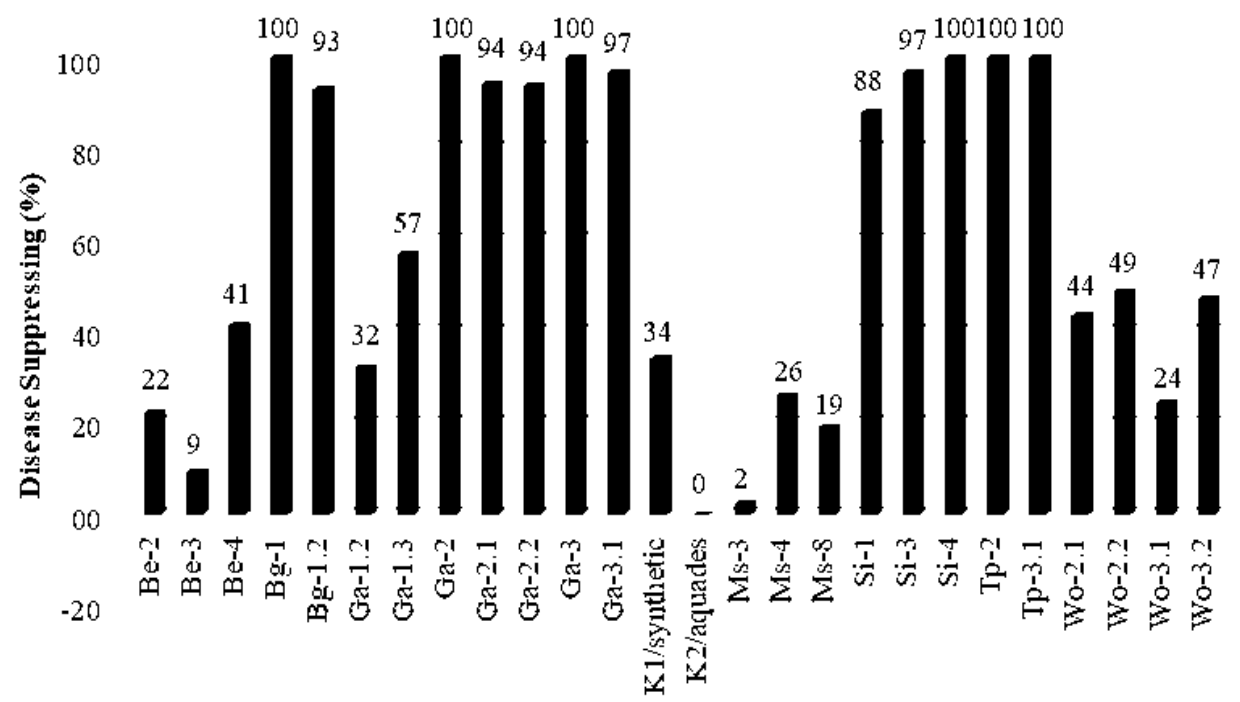

Treatment

Figure 1. Percentage of downy mildew suppression in various treatments 
$-$

55 NR 114582.1:40-1196 Bacillus cereus ATCC 14579 16S ribosomal RNA partial sequence

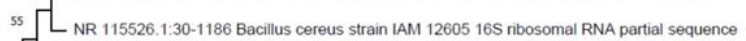
55 NR 112630.129-1185 Bacillus cereus strain NBRC 15305165 nibosomal RNA partial sequence

${ }_{55}^{55}$ NR 115714.1:50-1206 Bacillus cereus strain CCM 2010 16S nibosomal RNA partial sequence

55 NR 113266.1:30-1186 Bacillus cereus strain JCM 215216 r nibosomal RNA partial sequence

NR 074540.1.57-1213 Bacillus cereus ATCC 14579 16S ribosomal RNA (mA) partial sequence

NR 152692.1:50-1206 Bacillus wiedmannii strain FSL WB-0169 16S ribosomal RNA partial sequence

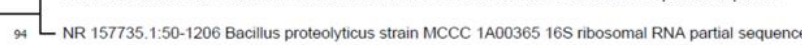

Bg-1

32 NR 157729.1:50-1206 Bacillus albus strain MCCC 1 A02 146 16S ribosomal RNA partial sequence

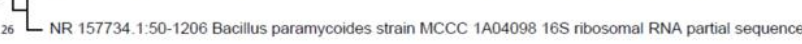

NR 116240.1:18-1173 Bacillus velezensis strain CBMB205 16S ribosomal RNA partial sequence

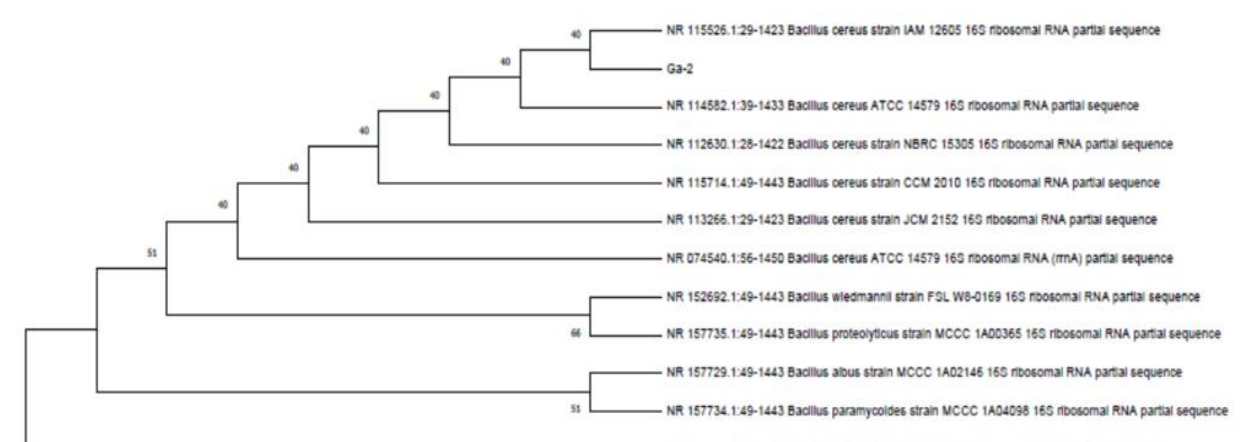

20.1.34-1453 Bacilius cereus strain TAM 12605165 nbosomal RNA partial sequenco

${ }_{30}{ }^{3 i-4}$

${ }^{39}$ NR 114582.1.44-1463 Bacillus cereus ATCC 14579 16S ribosomal RNA partial sequence

${ }^{39}$ NR 112630.1:33-1452 Bacillus cereus strain NBRC 15305 165 ribosomal RNA partial sequence

NR 115714.1:54-1473 Bacillus cereus strain CCM 2010 16S ribosomal RNA partial sequence

NR 113266.1:34-1453 Bacillus cereus strain JCM 2152165 ribosomal RNA partial sequence

NR 074540.1:161-1480 Bacillus cereus ATCC 14579 16S nibosomal RNA (mA) partial sequence

NR 152692.154-1473 Bacillus wiedmannii strain FSL W8-0169 16S nibosomal RNA partial sequence

${ }_{42} 2$ NR 157735.1:54-1473 Bacillus proteolyticus strain MCCC 1 A00365 165 r ribosomal RNA partial sequence NR 157729.154-1473 Bacillus albus strain MCCC 1 A02146 16S ribosomal RNA partial sequence

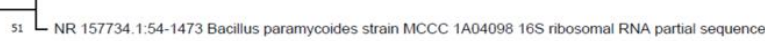

${ }_{55}$ NR 114582.139-1066 Bacillus cereus ATCC 14579 16S nibosomal RNA partial sequence

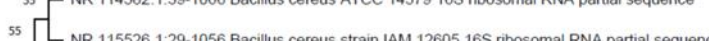
55 NR 112630.1:28-1055 Bacillus cereus strain NBRC 15305165 ribosomal RNA partial sequence 55 NR 115714.1.49-1076 Bacillus cereus strain CCM 201016 S ribosomal RNA partial sequence NR 113266.1:29-1056 Bacillus cereus strain JCM 2152 16S nibosomal RNA partial sequence - NR 074540.1:56-1083 Bacillus cereus ATCC 14579 16S nbosomal RNA (mA) partial sequence NR 152692: 1:49-1076 Bacillus wiedmanni strain FSL W8-0169 16S nibosomal RNA partial sequence $\Gamma^{\mathrm{Ga}-3}$

43 NR 157734.1:49-1076 Bacillus paramycoides strain MCCC 1A04098 16S ribosomal RNA partial sequence NR 157729 1-49-1076 Bacillus abuus strain MCCC 1 A02146 165 ribosomal RNA partial seoveree NR 027552:1:39-1064 Bacillus subtills strain DSM 10 16S nibosomal RNA partial sequence

\section{D}

${ }^{100}$ - NR 041715.1:1-1458 Pseudomonas stutzeri ATCC 17588 LMG $1119916 S$ ribosomal RNA partial sequence ${ }_{100}^{100}[$ NR 118798.1:1-1456 Pseudomonas stutzeri strain CCUG 11256 16S ribosomal RNA partial sequence 100 NR 113652.1:1-1462 Pseudomonas stutzeri strain NBRC 14165 16S ribosomal RNA partial sequence ${ }^{100}$ L NR 116489.1:1-1495 Pseudomonas stutzeri strain VKM B-975 165 r ribosomal RNA partial sequence

61 NR 103934.2:8-1508 Pseudomonas stutzeri ATCC 17588 LMG 11199 16S ribosomal RNA complete sequence NR 148295.11-1458 Pseudomonas songnenensis strain NEAU-ST5-5 16S ribosomal RNA partial sequence NR 133828 1:1-1498 Pseudomonas kunmingensis strain HL22-2 16S ribosomal RNA partial sequence ${ }_{34}\left[\begin{array}{l}\text { NR 133828.1:1-1498 Pseudomonas kunmingensis strain HL22-2 } 16 S \text { ribosomal RNA partial sequence } \\ \text { NR 115115.11-1488 Pseudomonas chloritidismutans strain AW-1 16S ribosomal RNA partial sequence }\end{array}\right.$ NR 115115.11-1488 Pseudomonas chlortidismutans strain AW-1 16 r ribosomal RNA partial sequence NR 135703.1:-1513 Pseudomonas guariconensis strain PCAVU11 $16 S$ ribosomal RNA partial sequ NR 114041.1:1-1463 Pseudomonas oryzihabitans strain NBRC 102199 16S ribosomal RNA partial sequence Tp-2

$\mathbf{E}$

Figure 2. Phylogenetic trees of Bg-1 (A), Si-4 (B), Ga-2 (C), Ga-3 (D), Tp-3.1 (E), and Tp-2 (F), and group-with (group ) trees his relatives 
Treatment of bacterial isolates can suppress DM infection in corn plants. The effective suppression of DM by isolates of $\mathrm{Bg}-1, \mathrm{Ga}-2, \mathrm{Ga}-3, \mathrm{Si}-4, \mathrm{Tp}-2$ and $\mathrm{Tp}-3.1$ was up to $100 \%$ at 35 DAP. The DM pressure was related to the ability of a bacterium to colonize the leaves and produce secondary metabolic compounds that can protect plants from pathogens. This similar result was obtained by Suryadi et al. (2013) that metabolic products produced by bacteria can inhibit disease incidence in plants. Bacteria can enter through the process of seed germination, secondary roots, stomata or injured leaves (Resti et al. 2013).

Downy mildew infection was negatively correlated with leaf chlorophyll. Plants treated with $\mathrm{Bg}-1, \mathrm{Ga}-2, \mathrm{Ga}-2.1$, Ga-3.1, Si-1, Si-3, Si-4, Tp-2, and Tp-3.1 bacterial isolates have lower DM incidence and high chlorophyll content than controls. DM infection decreases the chlorophyll content in corn plants. Hao et al. (2013) reported that DM infection reduced the photosynthetic rate and chlorophyll content in plants. The treatment of bacterial isolates showed that the results of the analysis were not significantly different from the control on aspects of plant height, but the fresh weight of the plants showed different results in each treatment of bacterial isolates, this was thought to be related to the different mechanism of action of each isolate shown by its ability to dissolve phosphate, potassium, and nitrogen-fixing. The results of the study by Manzoor et al. (2016) showed that the phosphate solvent bacteria were able to increase the biomass of corn plant and accumulation of phosphorus in plants. Pseudomonas fluorescens was capable of producing plant growthenhancing agents and antifungal substances, for the development of biological fertilizers and bioinoculants for food crops (Noori and Saud 2012).

Several bacterial genera have been used as biocontrol agents to control DM disease. The genus Bacillus and Serratia have been reported to control DM in cucumbers for controlling DM in cucumbers (Sun et al. 2013; Tesfagiorgis et al. 2014; Mohamed et al. 2016). Raj et al. (2017) reported that Bacillus strains can induce resistance in millet to control DM. Genus Pseudomonas has the potential to control downy mildew on mollusks and mustard greens (Elsharkawy et al. 2014; Damiri et al. 2017).

In conclusion, the $\mathrm{Bg}-1, \mathrm{Ga}-2, \mathrm{Ga}-3, \mathrm{Si}-4, \mathrm{Tp}-2$, and Tp-3.1 isolates were able to suppress DM infection and increase corn yield by up to $100 \%$. The selected bacterial isolates were Bacillus albus strain MCCC 1A02146, Bacillus cereus strain IAM 12605, Bacillus paramycoides strain MCCC 1A04098, Pseudomonas stutzeri strain CCUG 11256, Serratia marcescens subsp. sakuensis strain KRED. These isolates can be used as PGPR and biological pesticides to control downy mildew disease on corn.

\section{ACKNOWLEDGEMENTS}

The authors would like to thank Dr. Muhammad Azrai, Director of ICERI for essential help and support during this research work. All authors are main contributors.

\section{REFERENCES}

Damiri N, Umayah A, Agustin SE, Rahmiyah M. 2017. Effect of Pseudomonas spp on infection of Peronospora parasitica (Pers. Fr), the pathogen of downy mildew on Chinese cabbage. IOP Conf Ser Earth Environ Sci 102 (1): 012065. DOI: 10.1088/17551315/102/1/012065.

Elsharkawy MM, Kamel SM, El-Khateeb NMM. 2014. Biological control of powdery and downy mildews of cucumber under greenhouse conditions. Egypt J Biol Pest Control 24 (2): 301-308.

Hao Y, Wu C, Zhao D, Thung L, Yu Y, Fan H. 2013. Proteomic analysis of glucohexose induced resistance to downy mildew in Cucumis sativus. Aust J Crop Sci 7 (9): 1242.

Kaur P, Sivasithamparam K, Li H, Barbetti MJ. 2011. Pre-inoculation with Hyaloperonospora parasitica reduces incubation period and increases severity of disease caused by Albugo candida in a Brassica juncea variety resistant to downy mildew. J General Plant Pathol 77 (2): 101-106. DOI: 10.1007/s10327-011-0293-2.

Lantican DV, Canama AO, Malijan AKB, Pascual CB, Quilloy RB, Manguiat PH, Galvez H.F. 2013. Optimization of QTL region for downy mildew resistance corn. Philippine J Crop Sci 38 (suppl 1): 93.

Lukman R, Afifuddin A, Lübberstedt T. 2016. Tracing the signature of Peronosclerospora maydis in maize seeds. Australasian Plant Pathol 45 (1): 73-82. DOI: 10.1007/s13313-015-0390-3.

Manzoor M, Abbasi MK, Sultan T. 2016. Isolation of phosphate solubilizing bacteria from maize rhizosphere and their potential for rock phosphate solubilization-mineralization and plant growth promotion. Geomicrobiology J 34 (1): 81-95. DOI: 10.1080/01490451.2016.1146373

Meena VS, Maurya BR, Verma JP, Aeron A, Kumar A, Kim K, Bajpai VK. 2015. Potassium solubilizing rhizobacteria (KSR): isolation, identification, and K-release dynamics from waste mica. Ecol Eng 81: 340-347. DOI: 10.1016/j.ecoleng.2015.04.065.

Mohamed A, Hamza A, Derbalah A. 2016. Recent approaches for controlling downy mildew of cucumber under greenhouse conditions. Plant Protect Sci 52 (1): 1-9. DOI: 10.17221/63/2015-PPS.

Mota MS, Gomes CB, Souza Júnior IT, Moura AB. 2017. Bacterial selection for biological control of plant disease: criterion determination and validation. Brazilian J Microbiol 48 (1): 62-70. DOI: 10.1016/j.bjm.2016.09.003.

Noori MS, Saud HM. 2012. Potential plant growth-promoting activity of Pseudomonas sp. isolated from paddy soil in Malaysia as biocontrol agent. J. Plant Pathol Microb 3 (2): 1-4. DOI: 10.4172/21577471.1000120 .

Pudjiwati EH, Kuswanto K, Basuki N, Sugiharto AN. 2013. Path analysis of some leaf characters related to downy mildew resistance in maize. Agrivita J Agric Sci 35 (2): 167-173. DOI: 10.17503/Agrivita-201335-2-p167-173.

Raj SN, Lavanya SN, Amruthesh KN, Niranjana SR, Reddy MS, Shetty HS. 2012. Histo-chemical changes induced by PGPR during induction of resistance in pearl millet against downy mildew disease. Biol Control 60 (2): 90-102. DOI: 10.1016/j.biocontrol.2011.10.011.

Resti Z, Habazar T, Putra DP. 2013. Skrining dan identifikasi isolat bakteri endofit untuk mengendalikan penyakit hawar daun bakteri pada bawang merah. J. Hama dan Penyakit Tumbuhan Tropika 13 (2): 167-178. [Indonesian]

Sekarsari A, Prasetyo RJ, Maryono T. 2013. Pengaruh beberapa fugisida nabati terhadap keterjadian penyakit bulai pada tanaman jagung manis. J. Agrotek Tropika 1 (1): 98-101. [Indonesian]

Souza RD, Ambrosini A, Passaglia LM. 2015. Plant growth-promoting bacteria as inoculants in agricultural soils. Genet Mol Biol 38 (4): 401-419. DOI: 10.1590/S1415-475738420150053.

Sun ZB, Yuan XF, Zhang H, Wu LF, Liang C, Feng YJ. 2013. Isolation, screening and identification of antagonistic downy mildew endophytic bacteria from cucumber. Eur J Plant Pathol 137 (4): 847857. DOI: 10.1007/s10658-013-0293-5.

Suryadi Y, Priyatno TP, Samudra I, Susilowati DN, Patricia, Irawati W. 2013. Karakterisasi dan identifikasi isolat bakteri endofitik penghambat jamur patogen padi. Buletin Plasma Nutfah 19 (1): 2532. [Indonesian]

Tariq M, Noman M, Ahmed T, Hameed A, Manzoor N, Zafar M. 2017. Antagonistic features displayed by plant growth-promoting rhizobacteria (PGPR): a review. J Plant Sci and Phytopathol 1: 38-43. DOI: $10.29328 /$ journal.jpsp.1001004. 
Tesfagiorgis HB, Laing MD, Annegarn HJ. 2014. Evaluation of biocontrol agents and potassium silicate for the management of powdery mildew of zucchini Biol Control 73. $8-15$ DOI: 10.1016/j.biocontrol.2014.03.010.
Zhang X, Zhou Y, Li Y, Fu X, Wang Q. 2017. Screening and characterization of endophytic bacillus for biocontrol of grapevine downy mildew. Crop Protect 96: 173-179. DOI: 10.1016/j.cropro.2017.02.018. 